\title{
Superselective pseudocontinuous arterial spin labeling in patients with meningioma: utility in prediction of feeding arteries and preoperative embolization feasibility
}

\author{
Dong Hyun Yoo, MD, ${ }^{1}$ Chul-Ho Sohn, MD, PhD, ${ }^{1}$ Young Dae Cho, MD, PhD, ${ }^{1}$ \\ Hyun-Seung Kang, MD, PhD, ${ }^{2}$ Chul-Kee Park, MD, PhD, ${ }^{2}$ Jin Wook Kim, MD, PhD, ${ }^{2}$ and \\ Jae Hyoung Kim, MD, PhD ${ }^{3}$ \\ Departments of ${ }^{1}$ Radiology and ${ }^{2}$ Neurosurgery, Seoul National University Hospital, Seoul; and ${ }^{3}$ Department of Radiology,
Seoul National University Bundang Hospital, Gyeonggi-do, Republic of Korea
}

\begin{abstract}
OBJECTIVE Superselective pseudocontinuous arterial spin labeling (ss-pCASL) is an MRI technique in which individual vessels are labeled to trace their perfusion territories. In this study, the authors assessed its merit in defining feeding vessels and gauging preoperative embolization feasibility for patients with meningioma, using digital subtraction angiography (DSA) as the reference method.

METHODS Thirty-one consecutive patients with meningiomas were prospectively recruited, each undergoing DSA (and embolization, if feasible) before resection. All ss-pCASL imaging studies were performed 1 day prior to DSA. Two neuroradiologists independently reviewed ss-pCASL images, rating the contribution of each labeled vessel to tumor blood supply as none, minor, or major. Two neuroradiologists also gauged the feasibility of embolization in each patient, based on ss-pCASL images. Interobserver and intermodality agreement were determined using Cohen's kappa statistic. The diagnostic performance of ss-pCASL was assessed in terms of discerning tumor blood supply and the potential for embolization.
\end{abstract}

RESULTS Interobserver agreement in the rating of blood supply by ss-pCASL was very good $(\kappa=0.817,95 \% \mathrm{Cl}$ $0.771-0.863$ ), and intermodality agreement (consensus ss-pCASL readings vs DSA findings) was good $(\kappa=0.688,95 \%$ CI 0.632-0.744). In delineating tumor blood supply, ss-pCASL showed high sensitivity (87.1\%) and specificity (87.2\%). The positive and negative predictive values for embolization feasibility were $85.2 \%$ and $100 \%$, respectively.

CONCLUSIONS In patients with meningiomas, feeding vessels are reliably predicted by ss-pCASL. This noninvasive approach, involving no iodinated contrast or radiation exposure, is particularly beneficial if there are no prospects of embolization.

https://thejns.org/doi/abs/10.3171/2020.7.JNS201915

KEYWORDS arterial spin labeling; digital subtraction angiography; embolization; meningioma; territorial imaging; oncology

$\mathrm{M}$ ENINGIOMa is the most frequently diagnosed primary intracranial tumor. ${ }^{1}$ Most are hypervascular, the usual feeding arteries being dural branches of the external carotid artery (ECA) such as the middle meningeal artery (MMA). Feeding arteries also originate from dural or pial branches of the internal carotid artery (ICA) or vertebral artery (VA). ${ }^{2}$ Although some have questioned the benefit of preoperative embolization, ${ }^{3}$ depriving tumors of blood supply through embolization increases the chances of complete resection and limits intraoperative blood loss. ${ }^{4-6}$ However, not all feeding arteries are safe and suitable for this purpose. Those arising from the ICA or VA carry a substantial risk of cerebral ischemic complications, and embolization of superficial arteries (i.e., superficial temporal artery [STA] or occipital artery [OA]) may result in scalp necrosis. ${ }^{7,8}$ Digital subtraction angiography (DSA) remains the gold standard modality for the delineation of feeding arteries, and only upon its completion is

ABBREVIATIONS ASL = arterial spin labeling; DSA = digital subtraction angiography; $E C A=$ external carotid artery; ICA = internal carotid artery; MMA = middle meningeal artery; NPV = negative predictive value; $\mathrm{OA}=$ occipital artery; PPV = positive predictive value; $S N R=$ signal-to-noise ratio; ss-pCASL = superselective pseudocontinuous ASL; STA = superficial temporal artery; TOF-MRA = time-of-flight magnetic resonance angiography; VA = vertebral artery.

SUBMITTED May 22, 2020. ACCEPTED July 1, 2020.

INCLUDE WHEN CITING Published online November 13, 2020; DOI: 10.3171/2020.7.JNS201915. 
the prospect of embolization ascertained. However, DSA is an invasive procedure involving iodinated contrast and radiation exposure, carrying its own risks. ${ }^{9}$

Arterial spin labeling (ASL) is an entirely noninvasive MRI technique used to assess cerebral circulation by magnetically labeling inflowing blood. ${ }^{10}$ The more recent introduction of vessel-selective ASL further enables the tracing of single-vessel perfusion territories by labeling individual arteries. ${ }^{11-13}$ A number of studies have shown the merit of this technique in certain cerebrovascular disorders such as arteriovenous malformations and carotid artery stenosis, ${ }^{14,15}$ but the few efforts to date exploring its applicability to meningioma have lacked full comparisons with DSA. ${ }^{16-18}$ The present study provides a comparative analysis of superselective pseudocontinuous ASL (ss-pCASL) ${ }^{12}$ and DSA in patients with meningiomas, each used to define feeding vessels and gauge preoperative embolization feasibility. We hypothesized that ss-pCASL would reliably predict feeding vasculature of meningiomas.

\section{Methods \\ Patient Population}

This study was approved by the Seoul National University Hospital IRB, with all participating patients providing written informed consent. We prospectively recruited consecutive patients who met the following criteria: 1) age > 18 years, 2) intracranial meningioma suspected by imaging study, and 3) DSA (with embolization, if feasible) and subsequent resection scheduled at Seoul National University Hospital. In our institution, not all patients with meningioma were consulted for DSA. The need for DSA and embolization was defined by the neurosurgeons in charge of the surgery. Exclusion criteria were as follows: 1) uninterpretable MRI data due to artifacts, 2) previous history of open cranial surgery, 3) neurological disorders (aside from meningioma), or 4) histological evidence of tumors other than meningioma.

\section{MRI Studies}

All ss-pCASL imaging was performed 1 day prior to DSA using an Ingenia 3.0T MRI scanner (Philips Healthcare). Labeling areas were planned using time-of-flight magnetic resonance angiography (TOF-MRA), extending from the carotid artery bifurcation to the base of the skull. Potential labeling targets were the 5 major cervical arteries: both ICAs, both ECAs, and dominant- or lesionside VA. However, vessels were selectively labeled for sspCASL in each patient according to tumor location. At the investigator's discretion, vessels with little possibility of supplying the tumor were not labeled for image acquisition. For example, right ICA-labeled images were not pursued in a patient with left temporal convexity meningioma. All ss-pCASL images were acquired using the following parameters: field of view $240 \times 240 \times 96 \mathrm{~mm}^{3}$, voxel size $2.75 \times 2.75 \times 6 \mathrm{~mm}^{3}$, flip angle $90^{\circ}$, single-shot echo-planar imaging acquisition $(\mathrm{TR} / \mathrm{TE}=4265 / 9.7 \mathrm{msec}$, number of excitations $=1$ ), labeling duration 1.8 seconds, postlabeling delay 2.0 seconds, and labeled image slices = 16 . The scan time was approximately 3 minutes per vessel, with a maximum of approximately 15 minutes required per patient. The images were acquired in real time, and no postprocessing on a dedicated workstation was required.

\section{DSA and Embolization}

After puncture of the femoral artery and insertion of a 5-Fr arterial sheath, the VA on the dominant (larger) or lesion (in the case of a posterior fossa tumor) side, both ICAs, and both ECAs were selectively catheterized. If, in the presence of a hypervascular tumor stain, embolization was deemed safe and productive, a microcatheter was advanced to the presumptive feeding artery. Angiography was then performed via a microcatheter to replicate the tumor blush and exclude a potentially dangerous anastomosis. Polyvinyl alcohol particles were used for embolization.

\section{Analysis of ss-pCASL Technique}

Two readers (D.H.Y. and C.H.S.) experienced in ASL imaging and interventional neuroradiology (5 and 15 years, respectively) and blinded to DSA images independently reviewed the ss-pCASL images. The images from each patient were thoroughly anonymized, and then the images were classified and loaded in separate files with a description on which vessel was labeled. Initially, each labeled vessel was rated according to the extent of tumor blood supply as none, minor, or major. Minor supply vessels were those with ASL signals so inconsequential that embolization, even if technically feasible, would not effectively achieve tumor devascularization. Interobserver agreement was calculated using Cohen's kappa statistic, interpreted as follows: $<0.20=$ poor, $0.21-0.40=$ fair, $0.41-0.60=$ moderate, $0.61-0.80=$ good, $0.81-0.90=$ very good, and $>0.90=$ excellent. Final decisions on discrepant ratings were reached by consensus. In each study patient, image quality was also graded as fair or good by consensus of both readers. Using consensus readings of ss-pCASL images, the two readers then predicted the feasibility of embolization in each patient. When major supply was identified on ECA-labeled images of one or both sides, the tumor was judged suitable for embolization.

\section{Comparison With DSA Studies}

All tumor signals from labeled vessels were validated against the hypervascularity manifested in corresponding DSA views. DSA images were reviewed, blinded to sspCASL image, and angiographic vascularity was rated in the same manner (none, minor, or major supply) as in sspCASL images. Intermodality agreement was also calculated using Cohen's kappa. The diagnostic performance of ss-pCASL in delineating tumor blood supply was calculated on a per vessel basis, whereas the gauging of embolization was calculated on a per patient basis. All statistical computations relied on standard software (SPSS version 25, IBM Corp.).

\section{Results}

Between September 2018 and September 2019, 34 patients were prospectively enrolled for this study. Two were poorly cooperative and were excluded due to unin- 
TABLE 1. Characteristics of the tumors $(N=31)$ in the study population

\begin{tabular}{cc}
\hline Characteristic & Value $(\%)$ \\
\hline Tumor diameter $(\mathrm{mm})$ & \\
\hline Mean max \pm SD & $18.1 \pm 13.4$ \\
\hline Range & $20(64.5)$ \\
\hline Location of the tumor & $4(12.9)$ \\
\hline Cerebral convexity & $2(6.5)$ \\
\hline Parasagittal & $2(6.5)$ \\
\hline Falx & $2(6.5)$ \\
\hline Sphenoid ridge & $1(3.2)$ \\
\hline Planum sphenoidale & \\
\hline Petroclival & $5(16.1)$ \\
\hline Clinical symptoms & $2(6.5)$ \\
\hline Hemiparesis & $4(12.9)$ \\
\hline Paresthesia & $2(6.5)$ \\
\hline Visual symptom & $4(12.9)$ \\
\hline Seizure & $6(19.3)$ \\
\hline Cognitive dysfunction & $2(6.5)$ \\
\hline Headache & $6(19.3)$ \\
\hline Others & $24(77.4)$ \\
\hline Asymptomatic & $7(22.6)$ \\
\hline Histologic classification &
\end{tabular}

terpretable ss-pCASL images. The other 32 were treated surgically, with 1 subsequently receiving a diagnosis of glioblastoma. Therefore, 31 patients (18 women and 13 men, mean age $58.9 \pm 13.4$ years, range $26-81$ years) with histologically proven meningioma were eligible for study. Characteristics of the tumors in this study population are summarized in Table 1.

Of the 155 vessels (i.e., 5 major cervical arteries in each of 31 patients) eligible for labeling, only 117 (21 right ICAs, 27 left ICAs, 30 right ECAs, 31 left ECAs, and 8 VAs) were actually labeled during ss-pCASL imaging and served for the final analysis. Among the rest, 36 were devoid of any blood supply to the tumor on DSA, and angiograms of the right ICA in 2 patients showed minor blood flow to contralateral convexity meningiomas.

Interobserver agreement in rating of blood supply on ss-pCASL was very good $(\kappa=0.817,95 \%$ CI $0.771-0.863)$. Consensus readings of ss-pCASL identified 16 major and 6 minor supply vessels originating from the ICA (either side) or VA, whereas 37 major and 8 minor supply vessels originated from the ECA (either side). Image quality was graded as good and fair in 19 and 12 patients, respectively. Intermodality agreement (consensus ss-pCASL readings vs DSA findings) was good $(\kappa=0.688,95 \%$ CI $0.632-$ 0.744 ; Table 2). High sensitivity $(87.1 \%$, $95 \%$ CI $77.0 \%-$ $94.0 \%)$ and specificity $(87.2 \%, 95 \%$ CI $74.3 \%-95.2 \%)$ were observed in defining tumor blood supply (minor or major) by ss-pCASL.

Consensus decisions on tumor embolization yielded
TABLE 2. Intermodality agreement for rating blood supply

\begin{tabular}{crrrr}
\hline & \multicolumn{3}{c}{ DSA } & \\
\cline { 2 - 4 } Rating & None & Minor & Major & Total \\
\hline ss-pCASL & & & & \\
\hline None & 41 & 6 & 3 & 50 \\
\hline Minor & 5 & 6 & 3 & 14 \\
\hline Major & 1 & 4 & 48 & 53 \\
\hline Total & 47 & 16 & 54 & \\
\hline
\end{tabular}

Intermodality agreement was good $(\kappa=0.688,95 \% \mathrm{Cl} 0.632-0.744)$. Sensitivity was $87.1 \%(95 \% \mathrm{Cl} 77.0 \%-94.0 \%)$ and specificity was $87.2 \%(95 \% \mathrm{Cl}$ $74.3 \%-95.2 \%)$.

"feasible" in 27 and "infeasible" in 4 patients. All 4 patients considered unfit underwent DSA only (negative predictive value $[\mathrm{NPV}]=100 \%$; Supplementary Figure), with 23 of 27 patients judged as suitable proceeding to preoperative embolization (positive predictive value [PPV] = $85.2 \%, 95 \%$ CI $83.1 \%-87.1 \%$ ). Tumor embolization proved unacceptable in 3 patients whose main feeders, despite originating from the ECA, were either the STA or OA. In 1 patient, although bilateral MMAs were supplying the tumor, embolization was overruled due to small-sized feeding arteries and the faintness of tumor blush. There were no adverse events associated with DSA or embolization. Representative images from illustrative cases are shown in Figs. 1-3.

\section{Discussion}

To our knowledge, this study is the first to use DSA studies to fully corroborate vessel-selective ASL images of patients with meningiomas. Our data showed good intermodality agreement $(\kappa=0.688)$, with ss-pCASL performing well (sensitivity $=87.1 \%$, specificity $=87.2 \%$ ) in delineating tumor blood supply and in gauging embolization feasibility (PPV $=85.2 \%, \mathrm{NPV}=100 \%)$.

Previous studies involving intracranial tumor diagnostics have readily demonstrated the utility of ASL. ${ }^{19-21}$ In patients with meningiomas, ASL images were found to reflect vascular density and facilitate histopathological differentiation. ${ }^{22-24}$ Other researchers have also reported that ASL helped define angiographic vascularity, thereby aiding in the selection of candidates for preoperative embolization. ${ }^{25,26}$ Conventional ASL has its own merits as it produces more consistent images with less acquisition time and less artifact because there is no need for labeling of selected arteries. In addition, quantitative measurement of cerebral blood flow is possible on conventional ASL images. However, conventional ASL images acquired by nonselective labeling in the cervical plane do not provide information on the origins of feeding arteries. Even if hypervascularity is documented on conventional ASL, those with dominant pial supply would not be amenable to embolization. The definite advantage of ss-pCASL lies in its ability to distinguish the origin of feeding arteries, which may assist in selecting suitable candidates for effective embolization. Even in circumstances in which embolization is not planned, neurosurgeons can obtain preoperative 

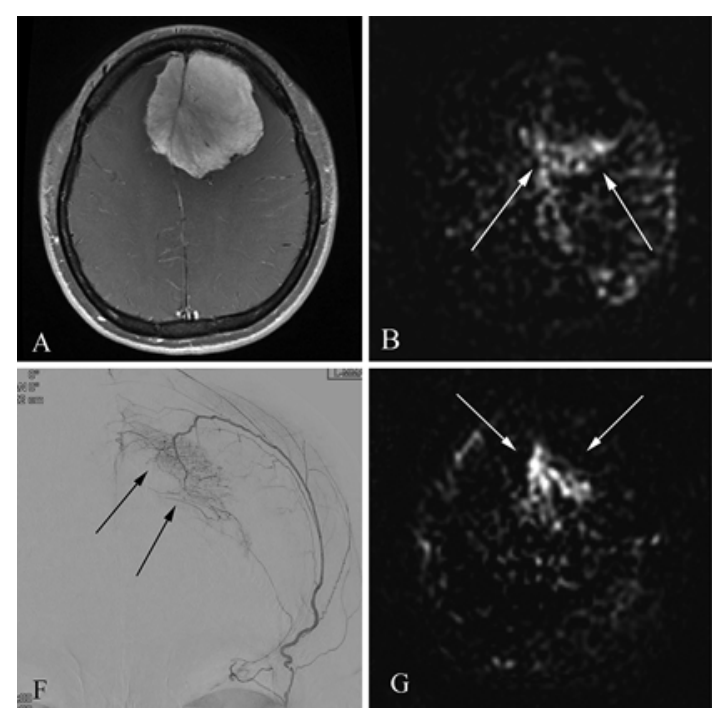
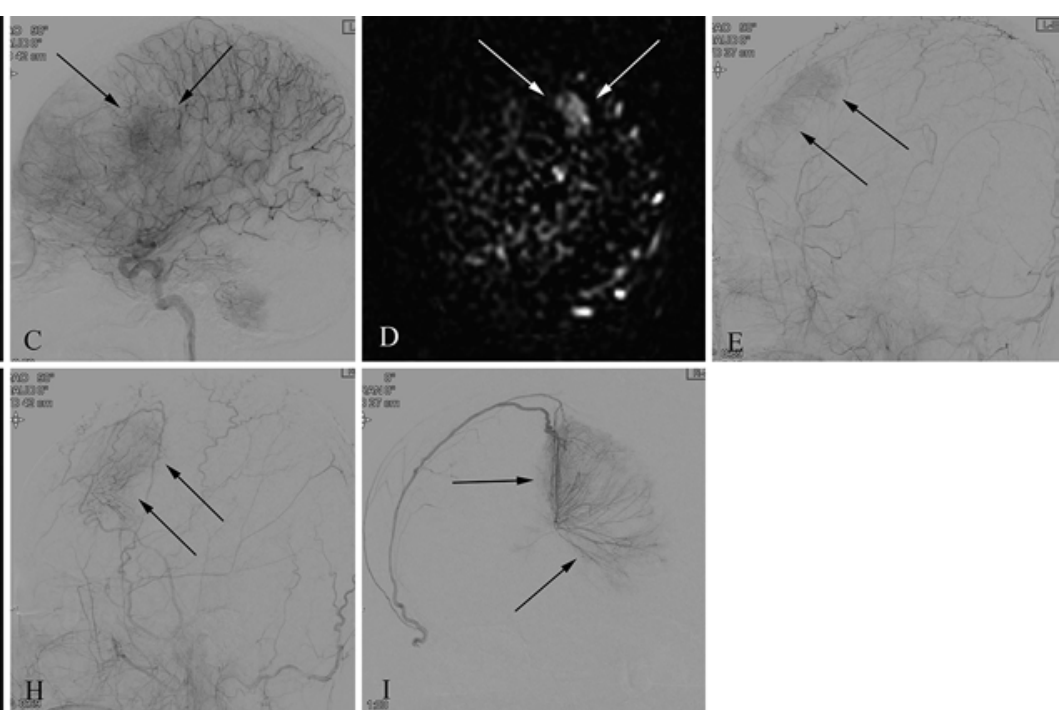

FIG. 1. Images obtained in a 35-year-old woman presenting with headache. A: Contrast-enhanced T1-weighted MR image of an anterior falcine meningioma (maximum diameter, $70 \mathrm{~mm}$ ). B: Left ICA-labeled ss-pCASL image of the posterior part of the tumor showing hyperperfusion (arrows). C: DSA of left ICA (lateral projection), hypervascular stain (arrows) at corresponding area of tumor. D: Left ECA-labeled ss-pCASL image of the anterolateral part of the tumor showing hyperperfusion (arrows). E and F: DSA of left ECA (lateral projection) and selective DSA of left MMA (anterior projection), hypervascular stain (arrows) at corresponding area of tumor. G: Right ECA-labeled ss-pCASL image of the posteromedial part of the tumor showing hyperperfusion (arrows). $\mathrm{H}$ and I: DSA of right ECA (lateral projection) and selective DSA of right MMA (anterior projection), hypervascular stain (arrows) at corresponding area of tumor. Bilateral MMA embolizations were performed.
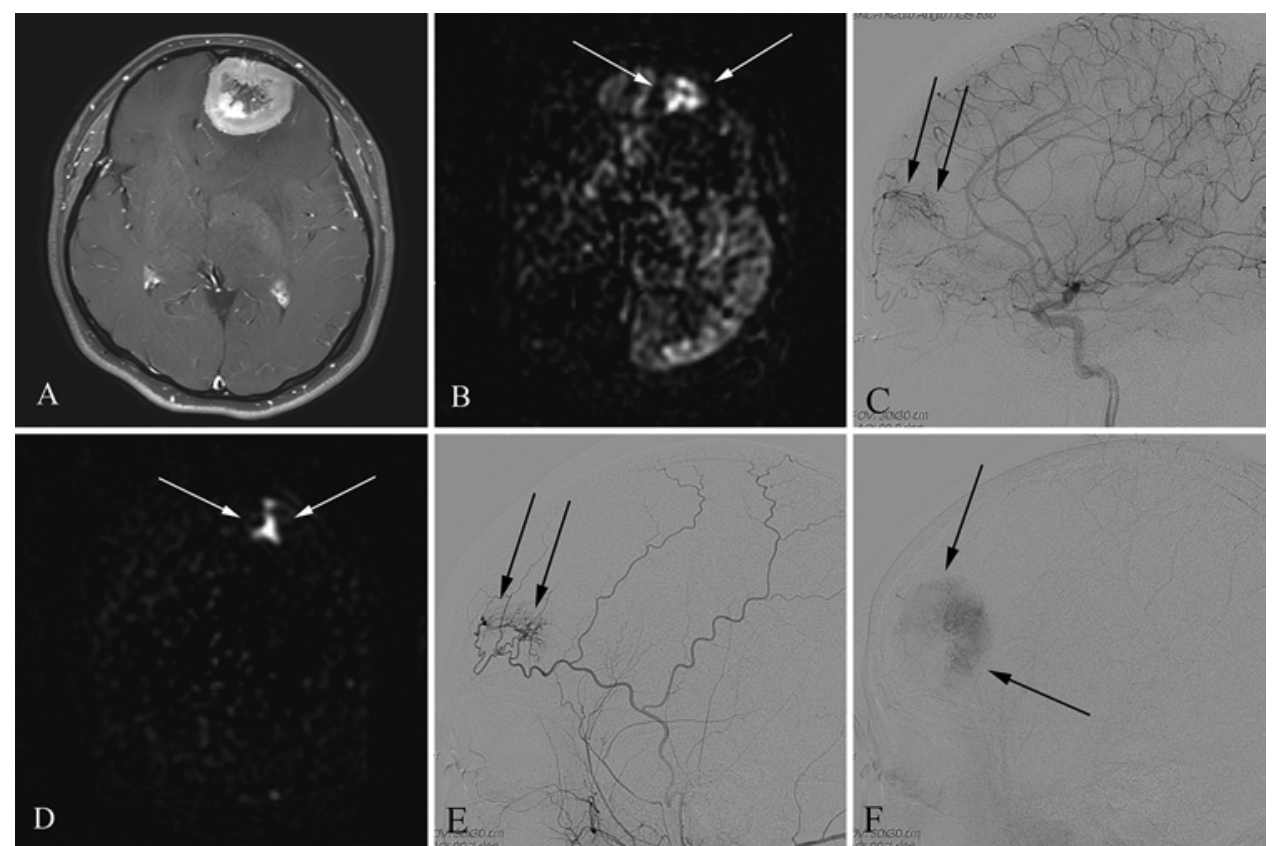

FIG. 2. Images from a 49-year-old woman presenting with seizure. A: Contrast-enhanced T1-weighted MR image of a left frontal convexity meningioma (maximum diameter, $42 \mathrm{~mm}$ ). B: Left ICA-labeled ss-pCASL image of the anteroinferior part of the tumor showing hyperperfusion (arrows). C: DSA of the left ICA (lateral projection), hypervascular stain (arrows) from the ethmoidal branch of the ophthalmic artery at the corresponding area of tumor. D: Left ECA-labeled SS-pCASL image of the anterosuperior part of the tumor showing hyperperfusion (arrows), rated as major supply. E: DSA of left ECA (lateral projection), hypervascular stain (arrows) from the STA at corresponding area of the tumor. F: DSA (more delayed phase), hypervascular stain (arrows) shifted to the posterior aspect of the tumor. Embolization was abandoned to avoid skin necrosis after STA occlusion. 

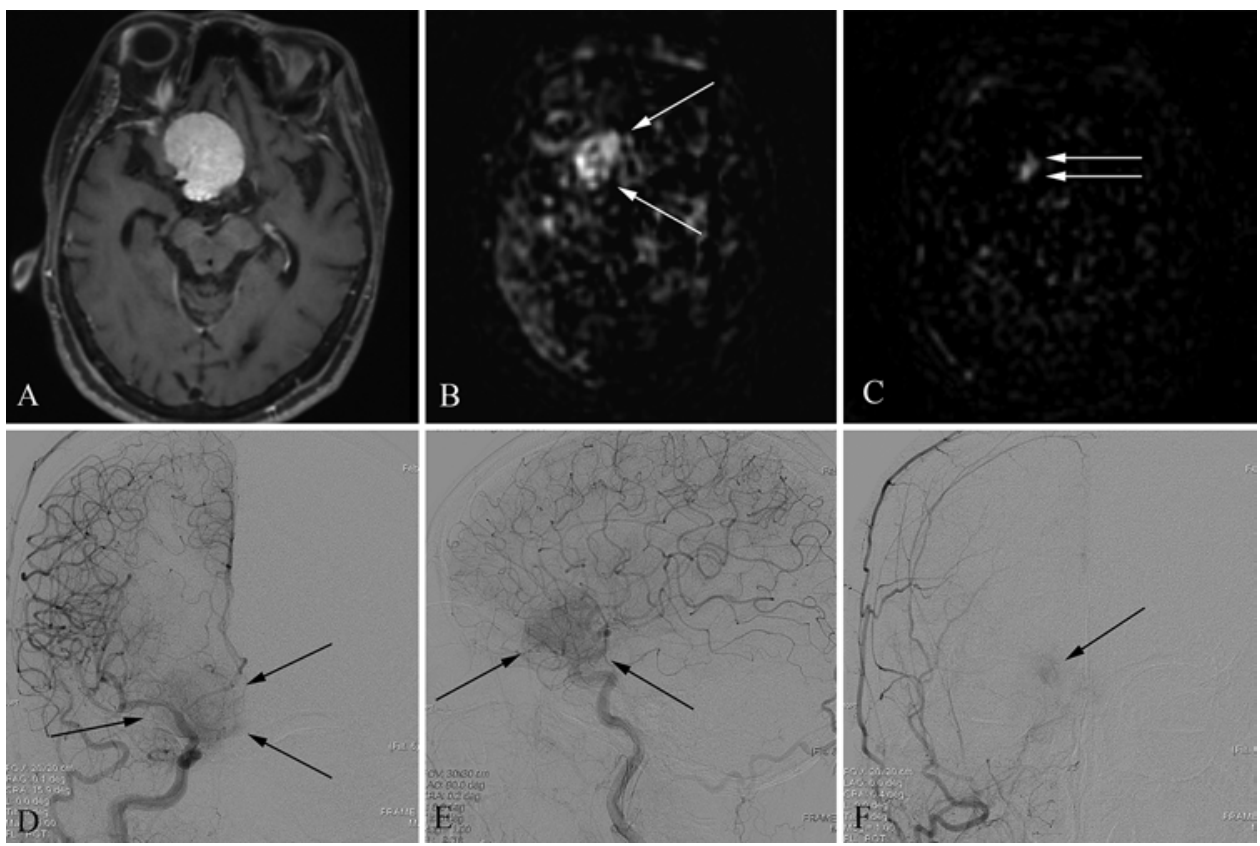

FIG. 3. Imaging in a 76-year-old woman presenting with diminished visual acuity. A: Contrast-enhanced T1-weighted MR image of a right planum sphenoidale meningioma (maximum diameter, $38 \mathrm{~mm}$ ). B: Right ICA-labeled ss-pCASL image of most of the tumor hyperperfused (arrows). C: Right ECA-labeled ss-pCASL image with limited focal hyperperfusion of the tumor (arrows), rated as minor supply. ICA-predominant vascular supply deemed unfit for embolization. D and E: DSA of right ICA showing hypervascular tumor stain (arrows) from a recurrent meningeal branch of the ophthalmic artery. F: DSA of right ECA (anterior projection) with focal hypervascular stain (arrows), presumably from an artery of the foramen rotundum. Embolization was not performed because arterial supply from the ECA branch was very small.

information on feeding vasculature, the presence and proportion of pial supply, without performing DSA.

The regional perfusion imaging of ss-pCASL is achieved through circular labeling of single arteries. These labeling spots, generated by rotating gradients with phase changes in radiofrequency pulses of pCASL labeling trains, are flexibly positioned, adapting to individual vessel geometries. ${ }^{12,15}$ As in previous studies, ${ }^{15,18}$ we successfully placed labeling spots on ICAs, ECAs, and the VA without much difficulty, even in patients with tortuous vessels. However, labeling is reliant on preacquired TOFMRA images, so even slight interim shifts in patient positions (between TOF-MRA and labeling) may interfere, leading to partial or no overlay of targeted arterial labeling spots. Inefficient arterial labeling of this sort results in a suboptimal signal-to-noise ratio (SNR). Moreover, images may be corrupted by signal contamination from nontargeted arteries, given the close proximity of the ICA and ECA. In brief, improper labeling may falsely depict tumor vascular supply, causing over- or underestimates.

In our patients, there were 12 underestimates (lower rating) and 10 overestimates (higher rating) of tumor vascular supply by ss-pCASL compared to DSA (Table 2). Underestimation was, in part, due to low SNRs obscuring fringe hyperperfusion of tumors (especially in ICAlabeled images), viewed as minor supply during DSA. Background noise or signal contamination also mimicked tumor vascularity on occasion, causing overestimation. Signal artifact induced by eyeball movement was yet another impediment to image interpretation. ${ }^{27}$ Intermodality agreement was good $(\kappa=0.772,95 \%$ CI $0.709-0.835)$ in 19 patients with good image quality, whereas it was moderate $(\kappa=0.551,95 \%$ CI $0.450-0.602)$ in 12 patients with fair image quality. These data imply that optimal imaging is crucial for the accurate definition of vessels feeding meningiomas. We were therefore compelled to exclude a pair of patients who constantly moved, undermining our repeated attempts at labeled image acquisition.

Apart from reduced image quality through movement, other factors were contemplated to explain discrepant outcomes of ss-pCASL and DSA studies. As shown in Fig. 2, insufficient postlabeling delays may falsely reduce areas of true hypervacularity in ASL images, fueling underestimates. Discrepancies may similarly arise when estimating visible tumor sizes and rating tumor blood supplies in each modality. Unlike biplanar 2D DSA projections, sspCASL images are analyzed via scrolling axial views, so perceived sizes may differ. Upon retrospective review of discordant ratings, departures typically arose when deciding if smaller fractions of visible tumors denoted minor or major arterial supply.

According to our data, ss-pCASL is a conclusive means of excluding tumors that are not amenable to embolization. However, embolization was abandoned in 4 of 27 meningiomas deemed acceptable for embolization by ss-pCASL. The chief culprits were tumors primarily supplied by scalp arteries through transosseous branches. A previous study has shown that primary feeder vessels are reliably identified by unenhanced MRA in 3D.$^{28}$ Hence, adjunctive MRA may have additive value in gauging the 
feasibility of embolization. It is also worth noting that our policy of leaving pial feeders or superficial arteries untouched for fear of complications is not a standard of care. Other dedicated colleagues have certainly performed otherwise and described their work. ${ }^{6,29}$

Several limitations of our study must be addressed. Due to limited resources and our reluctance to tax patient compliance, we did not acquire labeled images of all 5 major cervical arteries in every study patient. Considering the high specificity $(87.2 \%)$ shown by ss-pCASL, inclusion of all vessels would likely have strengthened our results. As a supplemental exercise, we designated all unlabeled arteries as nonsupply vessels, to reflect combined performance of ss-pCASL and investigator input. Again, intermodality agreement was good $(\kappa=0.727,95 \%$ CI $0.679-0.775)$, with high sensitivity (84.7\%) and specificity (92.8\%) for defining tumor blood supply.

Second, in the absence of volumetric computations, our subjective and inconsistent visual rating of minor versus major tumor supply was problematic. Lastly, the notion of DSA as the gold standard is also subject to debate. Various factors may be attributable to acquisition of suboptimal DSA images. At times, small areas of subtle tumor hypervascularity were not readily discernible from brain parenchymal stain or overlapping vessels on 2D images. These matters were exacerbated by patient movement or catheter-induced spasm of targeted arteries. In reviewing a few supposed false-positive cases, suspicious tumor vascularity (previously unnoticed in blind readings) was observed on DSA images.

Despite the above shortcomings, ss-pCASL has true potential as a viable substitute for DSA in patients with meningiomas. Future directions may include superselective labeling of smaller arterial branches such as the MMA or STA. Further research incorporating conventional ASL and MRA with ss-pCASL may also help better assess the feasibility of embolization in this setting.

\section{Conclusions}

Our findings suggest that vessels feeding meningiomas are reliably delineated using the ss-pCASL technique. Furthermore, the screening of meningiomas by ss-pCASL seems particularly beneficial if embolization is not plausible. In such circumstances, DSA may be entirely avoided or limited to selected vessels, reducing radiation exposure, use of contrast agents, and procedural risks.

\section{Acknowledgments}

The Seoul National University Hospital (SNUH) Medical Research Collaborating Center contributed to the review of statistical analysis. This study was supported by grant no. 0420180770 from the SNUH Research Fund.

\section{References}

1. Wiemels J, Wrensch M, Claus EB. Epidemiology and etiology of meningioma. J Neurooncol. 2010;99(3):307-314.

2. Bitzer M, Wöckel L, Luft AR, et al. The importance of pial blood supply to the development of peritumoral brain edema in meningiomas. J Neurosurg. 1997;87(3):368-373.

3. Bendszus M, Rao G, Burger R, et al. Is there a benefit of preoperative meningioma embolization? Neurosurgery. 2000;47(6):1306-1312.

4. Dowd CF, Halbach VV, Higashida RT. Meningiomas: the role of preoperative angiography and embolization. Neurosurg Focus. 2003;15(1):E10.

5. Shah AH, Patel N, Raper DM, et al. The role of preoperative embolization for intracranial meningiomas. J Neurosurg. 2013;119(2):364-372.

6. Raper DM, Starke RM, Henderson F Jr, et al. Preoperative embolization of intracranial meningiomas: efficacy, technical considerations, and complications. AJNR Am J Neuroradiol. 2014;35(9):1798-1804.

7. Adler JR, Upton J, Wallman J, Winston KR. Management and prevention of necrosis of the scalp after embolization and surgery for meningioma. Surg Neurol. 1986;25(4):357-360.

8. Dubel GJ, Ahn SH, Soares GM. Contemporary endovascular embolotherapy for meningioma. Semin Intervent Radiol. 2013;30(3):263-277.

9. Willinsky RA, Taylor SM, TerBrugge K, et al. Neurologic complications of cerebral angiography: prospective analysis of 2,899 procedures and review of the literature. Radiology. 2003;227(2):522-528.

10. Haller S, Zaharchuk G, Thomas DL, et al. Arterial spin labeling perfusion of the brain: emerging clinical applications. Radiology. 2016;281(2):337-356.

11. Wong EC. Vessel-encoded arterial spin-labeling using pseudocontinuous tagging. Magn Reson Med. 2007;58(6):10861091.

12. Helle M, Norris DG, Rüfer S, et al. Superselective pseudocontinuous arterial spin labeling. Magn Reson Med. 2010;64(3):777-786.

13. Lindner T, Helle M, Jansen O. A short introduction to arterial spin labeling and its application to flow territory mapping. Clin Neuroradiol. 2015;25(suppl 2):211-218.

14. Yu SL, Wang R, Wang R, et al. Accuracy of vessel-encoded pseudocontinuous arterial spin-labeling in identification of feeding arteries in patients with intracranial arteriovenous malformations. AJNR Am J Neuroradiol. 2014;35(1):65-71.

15. Richter V, Helle M, van Osch MJ, et al. MR imaging of individual perfusion reorganization using superselective pseudocontinuous arterial spin-labeling in patients with complex extracranial steno-occlusive disease. AJNR Am J Neuroradiol. 2017;38(4):703-711.

16. Sasao A, Hirai T, Nishimura S, et al. Assessment of vascular supply of hypervascular extra-axial brain tumors with 3T MR regional perfusion imaging. AJNR Am J Neuroradiol. 2010;31(3):554-558.

17. Lu Y, Luan S, Liu L, et al. Evaluation of the applicability of territorial arterial spin labeling in meningiomas for presurgical assessments compared with 3-dimensional time-of-flight magnetic resonance angiography. Eur Radiol. 2017;27(10):4072-4081.

18. Jensen-Kondering U, Helle M, Lindner T, et al. Non-invasive qualitative and semiquantitative presurgical investigation of the feeding vasculature to intracranial meningiomas using superselective arterial spin labeling. PLoS One. 2019;14(4):e0215145.

19. Warmuth C, Gunther M, Zimmer C. Quantification of blood flow in brain tumors: comparison of arterial spin labeling and dynamic susceptibility-weighted contrast-enhanced MR imaging. Radiology. 2003;228(2):523-532.

20. Noguchi T, Yoshiura T, Hiwatashi A, et al. Perfusion imaging of brain tumors using arterial spin-labeling: correlation with histopathologic vascular density. AJNR Am J Neuroradiol. 2008;29(4):688-693.

21. Kong L, Chen H, Yang Y, Chen L. A meta-analysis of arterial spin labelling perfusion values for the prediction of glioma grade. Clin Radiol. 2017;72(3):255-261.

22. Kimura H, Takeuchi H, Koshimoto Y, et al. Perfusion 
imaging of meningioma by using continuous arterial spinlabeling: comparison with dynamic susceptibility-weighted contrast-enhanced MR images and histopathologic features. AJNR Am J Neuroradiol. 2006;27(1):85-93.

23. Koizumi S, Sakai N, Kawaji H, et al. Pseudo-continuous arterial spin labeling reflects vascular density and differentiates angiomatous meningiomas from non-angiomatous meningiomas. J Neurooncol. 2015;121(3):549-556.

24. Lu Y, Xiong J, Yin B, et al. The role of three-dimensional pseudo-continuous arterial spin labelling in grading and differentiating histological subgroups of meningiomas. Clin Radiol. 2018;73(2):176-184.

25. Yoo RE, Yun TJ, Cho YD, et al. Utility of arterial spin labeling perfusion magnetic resonance imaging in prediction of angiographic vascularity of meningiomas. J Neurosurg. 2016;125(3):536-543.

26. Mayercik V, Ma M, Holdsworth S, et al. Arterial spin-labeling MRI identifies hypervascular meningiomas. AJR Am J Roentgenol. 2019;213(5):1124-1128.

27. Wood ML, Henkelman RM. MR image artifacts from periodic motion. Med Phys. 1985;12(2):143-151.

28. Uetani H, Akter M, Hirai T, et al. Can 3T MR angiography replace DSA for the identification of arteries feeding intracranial meningiomas? AJNR Am J Neuroradiol. 2013;34(4):765-772.

29. Hirohata M, Abe T, Fujimura N, et al. Preoperative embolization of brain tumor with pial artery or dural branch of internal carotid artery as feeding artery. Interv Neuroradiol. 2006;12(1)(suppl 1):246-251.

\section{Disclosures}

The authors report no conflict of interest concerning the materials or methods used in this study or the findings specified in this paper.

\section{Author Contributions}

Conception and design: Sohn, Yoo, JH Kim. Acquisition of data: Yoo, Cho, Kang, Park, JW Kim. Analysis and interpretation of data: Sohn, Yoo, Cho, Kang. Drafting the article: Yoo. Critically revising the article: Sohn, Cho, Kang, Park, JW Kim, JH Kim. Reviewed submitted version of manuscript: all authors. Approved the final version of the manuscript on behalf of all authors: Sohn. Statistical analysis: Yoo. Administrative/technical/material support: Park, JW Kim. Study supervision: Sohn, JH Kim.

\section{Supplemental Information}

\section{Online-Only Content}

Supplemental material is available with the online version of the article.

Supplementary Figure. https://thejns.org/doi/suppl/10.3171/ 2020.7.JNS201915.

\section{Correspondence}

Chul-Ho Sohn: Seoul National University Hospital, Seoul, Korea. neurorad63@gmail.com. 